\title{
Contradiction of Modal Modification
}

\author{
Miloš Kosterec*
}

The theory of property modification studies the logic and semantics of such terms as fake banknote, former president, and skilled surgeon. Terms like fake, former, and skilled (among many others) are called property modifiers. In general, a property modifier combines with a property to make a new property. Supposedly, there are four main types of property modifiers: intersective, subsective, privative and modal. One way to model the semantic properties of a modifier is to specify the particular type of entailment it appears in. For example, in the case of an intersective modifier, if something is a grey elephant, we know that it is grey and that it is an elephant. Consider a subsective modifier like skilled. If we know that somebody is a skilled surgeon, then we know that he or she is a surgeon. Now consider a privative modifier like fake. If we know that something is a fake banknote, then we know that it is not a banknote.

Here, I discuss the specification as well as the provided explication of modal modifiers via entailments. I demonstrate that both the specification and the explication are contradictory. First, modal modifiers are specified as follows:

The unique feature, however, that modal modifiers have is that they oscillate between being subsective and being privative. So if the premise is that $a$ is an alleged terrorist, say, then it is logically

* Institute of Philosophy of the Slovak Academy of Sciences

Institute of Philosophy, Slovak Academy of Sciences, Klemensova 19, 81364 Bratislava, Slovak Republic

$\triangle$ milos.kosterec@gmail.com

(C) The Author. Journal compilation (C) The Editorial Board, Organon F. 
possible that $a$ be a terrorist and it is logically possible that $a$ not be a terrorist. (Jespersen and Carrara 2013, 563)

(Jespersen 2015) utilizes Transparent Intensional Logic when formally specifying the entailments that involve modal modification:

Modal. $\lambda w \lambda t\left[\left[{ }^{0} M_{m}{ }^{0} F\right]{ }^{0} a\right]$ entails

$\lambda w \lambda t\left[{ }^{0} \lambda w^{\prime}\left[{ }^{0} \lambda t^{\prime}\left[\left[\left[{ }^{0} M_{m}{ }^{0} F\right]_{w t}{ }^{0} a\right] \rightarrow\left[{ }^{0} F_{w^{\prime} t^{\prime}}{ }^{0} a\right]\right]\right]\right.$

$\left.\wedge^{0} \lambda w^{\prime \prime}\left[{ }^{0} \lambda t^{\prime \prime}\left[\left[\left[{ }^{0} M_{m}{ }^{0} F\right] w t{ }^{0} a\right] \rightarrow\left[{ }^{0} N o n{ }^{0} F_{w^{\prime \prime} t^{\prime \prime}}{ }^{0} a\right]\right]\right]\right]$

(e.g. an alleged assassin is maybe an assassin). (Jespersen 2015,

$336-37)$

The elimination rule for modal modifier $M_{m}$ applied to property $f$ is then stated as follows (see Jespersen and Primiero 2013, 104):

$$
\frac{\left[\left[M_{m} f\right]_{w t} x\right]}{\lambda w^{\prime}\left[\lambda t^{\prime}\left[\left[\left[M_{m} f\right]_{w t} x\right] \rightarrow\left[f_{w^{\prime} t^{\prime}} x\right]\right]\right] \wedge \lambda w^{\prime \prime}\left[\lambda t^{\prime \prime}\left[\left[\left[M_{m} f\right]_{w t} x\right] \rightarrow \neg\left[f_{w^{\prime \prime} t^{\prime \prime}} x\right]\right]\right]}
$$

Now consider alleged discoverers of the highest prime number as an example of modal modification. The highest prime number cannot exist. Therefore, there are no such discoverers. This does not mean, however, that somebody, say Kurt Gödel, could not be alleged to be among such discoverers. But then, following both the informal and the formal specification of modal modifiers, it should be logically possible, i.e. there should be a possible world in which Kurt Gödel is one of the discoverers of the highest prime number. But surely there is no such world, because there cannot be such a number. Therefore, the general specification of modal modifiers leads to contradiction when applied to the data.

This generalizes to every use of modal modifiers with regard to properties that cannot be instantiated. Such a property may be the intension, e.g., of a contradictory property concept such as married bachelor. The contradiction need not always be present in the concept, however. Consider the property concept the necessarily empty property. In general, if $P$ stands for the necessarily empty property, $M$ is a modal modifier, and $k$ is an individual, then $\left[\begin{array}{ll}M & P\end{array}\right](\mathrm{k})$ leads to contradiction according to both the informal and the formal specification of modal modifiers. We can block the contradiction by stipulating that modal modifiers ought to be applied only to possibly non-empty properties. Such a stipulation is merely ad-hoc, however. 
Therefore, there is a need for the non-contradictory specification of modal modification. Perhaps switching from logical to epistemological possibility is in order, at least in the case of modal modifiers. (Jespersen and Primiero 2013) also seem to be suggesting non-factive approach to modal modifiers as a viable route of investigation:

The actual truth of $[M m F]$ a entails that one of two possibilities is realized: $a$ being an $F$; $a$ not being an $F$. Thus there is a striking similarity between modal modifiers and non-factive attitudes. (Jespersen and Primiero 2013, 98)

The link between modal modifiers and non-factive attitudes probably runs deeper than we let on in the present paper. [...] list of 'plain nonsubsective' (in effect, modal) modifiers/adjectives: potential, alleged, arguable, likely, predicted, putative, questionable, disputed. With the exception of potential, they all have something attitudinal about them. And all of those attitudes are nonfactive. A bold hypothesis would be that almost all modal modifiers are parasitic on non-factive attitudes. (Jespersen and Primiero 2013, 98 , footnote 10)

\section{Funding}

This paper was supported by VEGA Grant No. 2/0117/19 Logic, epistemology and metaphysics of fiction.

\section{References}

Jespersen, Bjorn, and Massimiliano Carrara. 2013. "A New Logic of Technical Malfunction." Studia Logica 101 (3): 547-81. https://doi.org/10.1007/s11225-0129397-8

Jespersen, Bjorn, and Giuseppe Primiero. 2013. "Alleged Assassin: Realist and Constructivist Semantics for Modal Modification." In Logic, Language, and Computation, edited by Guram Bazhanishvilli, Sebastian Löbner, Vincenzo Marra, and Frank Richter, 94-114. Berlin, Heidelberg: Springer-Verlag. https://doi.org/10.1007/978-3-642-36976-6_8 Jespersen, Bjorn. 2015. "Structured Lexical Concepts, Property Modifiers, and Transparent Intensional Logic." Philosophical Studies 172 (2): 321-45. https://doi.org/10.1007/s11098-014-0305-0 